\title{
COMMON FIXED POINT THEOREMS FOR COMPATIBLE MAPPINGS
}

\author{
KENAN TAŞ \\ Department of Mathematics \\ Abant Izzet Baysal University \\ Bolu, 14280, TURKEY \\ MUSTAFA TELCI \\ Department of Mathematics \\ Hacettepe University \\ Beytepe \\ Ankara 06532, TURKEY \\ BRIAN FISHER \\ Department of Mathematics and Computer Science \\ University of Leicester \\ Leicester, LE1 7RH, ENGLAND
}

(Received August 30, 1994 and in revised form December 6, 1994)

\begin{abstract}
By using a compatibility condition due to Jungck we establish some common fixed point theorems for four mappings on complete and compact metric spaces These results also generalize a theorem of Sharma and Sahu
\end{abstract}

KEY WORDS AND PHRASES. Common fixed point, commuting mappings, weakly commuting mappings and compatible mappings

1991 AMS SUBJECT CLASSIFICATION CODE. 54H 25

\section{INTRODUCTION.}

The following theorem was given by Sharma and Sahu [3]

THEOREM 1.1. Let $(X, d)$ be a complete metric space and let $S, T$ and $A$ be three continuous mappings of $X$ into itself satisfying the conditions

(i) $S A=A S, \quad T A=A T$,

(ii) $S(X) \subseteq A(X), \quad T(X) \subseteq A(X)$,

and the inequality

$$
\begin{aligned}
{[d(S x, T y)]^{2} \leq } & a_{1} d(A x, S x) d(A y, T y)+a_{2} d(A x, T y) d(A y, S x) \\
& +a_{3} d(A x, S x) d(A x, T y)+a_{4} d(S x, A y) d(T y, A y) \\
& +a_{5}[d(A x, A y)]^{2}
\end{aligned}
$$

for all $x, y$ in $X$, where $a_{\imath} \geq 0$ for $i=1,2,3,4,5, a_{1}+a_{4}+a_{5}<1$ and $2 a_{1}+3 a_{3}+2 a_{5}<2$, then $S, T$ and $A$ have a unique common fixed point in $X$

Unfortunately, the theorem as stated is incorrect First of all the only restriction on $a_{2}$ is that $a_{2} \geq 0 \quad$ This is clearly false In fact the inequality $a_{1}+a_{4}+a_{5}<1$ should have been $a_{2}+a_{4}+a_{5}<1$ This was only a typing error 
More seriously, on examination of the proof it is seen that the inequality $2 a_{1}+3 a_{3}+2 a_{5}<2$ was obtained by reading $\left[d\left(A x_{2 n}, A x_{2 n \cdot 2}\right)\right]^{2}$ as $\left[d\left(A x_{2 n+1}, A x_{2 n}\right]^{2} \quad\right.$ This inequality should have been $a_{1}+2 a_{3}+a_{5}<1$ The proof continues with a 'similarity ', but on following through it is seen that this time the inequality $a_{1}+2 a_{4}+a_{5}<1$ is required

\section{PRELIMINARIES.}

The following definitions were given by Sessa [2] and Jungck [1] respectively

DEFINITION 2.1. [2] Self-mappings $f$ and $g$ on a metric space $(X, d)$ are said to weakly commute if and only if $d(f g x, g f x) \leq d(f x, g x)$ for all $x$ in $X$

DEFINITION 2.2. [1] Self-mappings $f$ and $g$ on a metric space $(X, d)$ are said to be compatible if and only if whenever $\left\{x_{n}\right\}$ is a sequence in $X$ such that $\lim _{n \rightarrow \infty} f x_{n}=\lim _{n \rightarrow \infty} g x_{n}=t$ for some $t$ in $X$, then $\lim _{n \rightarrow \infty} d\left(f g x_{n}, g f x_{n}\right)=0$

Note that $f$ and $g$ commute, then it is easily seen that they weakly commute and if $f$ and $g$ weakly commute, then they are compatible However, weakly commuting mappings do not necessarily commute and compatible mappings do not necessarily weakly commute For examples, see [1] and [2]

PROPOSITION 2.1. [1] Let $f$ and $g$ be compatible self-mappings on a metric space $(X, d)$ with the properties

(i) if $f(t)=g(t)$, then $f g(t)=g f(t)$,

(ii) $\lim _{n \rightarrow \infty} f\left(x_{n}\right)=\lim _{n \rightarrow \infty} g\left(x_{n}\right)=t$

Then $\lim _{n \rightarrow \infty} g f\left(x_{n}\right)=f(t)$ if $f$ is continuous

We now prove the following theorem

THEOREM 2.1. Let $f$ and $g$ be self-mappings of the set $X=\left\{x, x^{\prime}\right\}$ with any metric $d$ If the range of $g$ contains the range of $f$, then the following statements are equivalent

(i) $f$ and $g$ commute,

(ii) $f$ and $g$ weakly commute,

(iii) $f$ and $g$ are compatible

PROOF. The implication that (i) $\Rightarrow$ (ii) $\Rightarrow$ (iii) is clear It is therefore sufficient to show that (iii) $\Rightarrow$ (i) The four self-mappings on $X$ are as follows

$$
A x=x, A x^{\prime}=x^{\prime}, \quad B x=B x^{\prime}=x, \quad C x=C x^{\prime}=x^{\prime}, \quad D x=x^{\prime}, \quad D x^{\prime}=x .
$$

We note that $A$ is the identity mapping on $X$ and since we only have to prove that compatible mappings commute, we may suppose that $f \neq g, f \neq A$ and $g \neq A$ Further, since $f(X) \subseteq g(X)$, we only have to consider the following two cases

$$
\begin{array}{lll}
\text { (a) } B=f, \quad D=g & \text { and } & \text { (b) } C=f, \quad D=g .
\end{array}
$$

If $f$ and $g$ are compatible and (a) holds, then $B x^{\prime}=D x^{\prime}$ implies that $B D x^{\prime}=D B x^{\prime}$ by Proposition 21 Consequently, $x=x^{\prime}$, a contradiction

Similarly, if (b) holds, then $D x=C x$ implies that $D C x=C D x$ by Proposition 2.1 and again $x=x^{\prime}$, a contradiction The conditions of the theorem can therefore hold only if either $f=g$ or $f=A$ or $g=A$ and the commutativity of $f$ and $g$ follows This completes the proof

\section{MAIN RESULTS.}

Let $A, B, S$ and $T$ be self-mappings of a metric space $(X, d)$ such that

$$
\begin{aligned}
& A(X) \subseteq T(X) \quad \text { and } \quad B(X) \subseteq S(X) \\
& {[d(A x, B y)]^{2} \leq } c_{1} \max \left\{[d(S x, A x)]^{2},[d(T y, B y)]^{2},[d(S x, T y)]^{2}\right\} \\
&+c_{2} \max \{d(S x, A x) d(S x, B y), d(A x, T y) d(B y, T y)\} \\
&+c_{3} d(S x, B y) d(T y, A x)
\end{aligned}
$$


for all $x, y$ in $X$, where $c_{1}, c_{2}, c_{3} \geq 0, c_{1}+2 c_{2}<1$ and $c_{1}+c_{3}<1$

Now let $x_{0}$ be an arbitrary point in $X$ Then since ( 31 l) holds, we can define a sequence

$$
\left\{y_{n}\right\}=\left\{A x_{0}, B x_{1}, A x_{2}, B x_{3}, \ldots, A x_{2 n}, B x_{2 n+1}, \ldots\right\}
$$

inductively such that $y_{2 n}{ }_{1}=T x_{2 n-1}=A x_{2 n-2}, y_{2 n}=S x_{2 n}=B x_{2 n}$ ifor $n=1,2, \ldots$

For simplicity, let $d_{n}=d\left(y_{n}, y_{n+1}\right)$ for $n=0,1,2, \ldots$ We need the following lemma for our main theorem

LEMMA 3.1. The sequence $\left\{y_{n}\right\}$ defined in (3 3 ) is a Cauchy sequence

PROOF. Using inequality ( 32 2) we have

$$
\begin{aligned}
d_{2 n+1}^{2}= & {\left[d\left(y_{2 n+1}, y_{2 n+2}\right)\right]^{2}=\left[d\left(A x_{2 n}, B x_{2 n+1}\right)\right]^{2} } \\
\leq & c_{1} \max \left\{\left[d\left(S x_{2 n}, A x_{2 n}\right)\right]^{2},\left[d\left(T x_{2 n+1}, B x_{2 n+1}\right)\right]^{2},\left[d\left(S x_{2 n}, T x_{2 n+1}\right)\right]^{2}\right\} \\
& +c_{2} \max \left\{d\left(S x_{2 n}, A x_{2 n}\right) d\left(S x_{2 n}, B x_{2 n+1}\right), d\left(A x_{2 n}, T x_{2 n+1}\right) d\left(B x_{2 n+1}, T x_{2 n+1}\right)\right\} \\
& \quad+c_{3} d\left(S x_{2 n}, B x_{2 n+1}\right) d\left(T x_{2 n+1}, A x_{2 n}\right) \\
\leq & c_{1} \max \left\{d_{2 n}^{2}, d_{2 n+1}^{2}\right\}+c_{2} d_{2 n} d\left(y_{2 n}, y_{2 n+2}\right) \\
\leq & c_{1} \max \left\{d_{2 n}^{2}, d_{2 n+1}^{2}\right\}+c_{2}\left[d_{2 n}^{2}+d_{2 n} d_{2 n+1}\right] \\
\leq & c_{1} \max \left\{d_{2 n}^{2}, d_{2 n+1}^{2}\right\}+c_{2}\left[\frac{3}{2} d_{2 n}^{2}+\frac{1}{2} d_{2 n+1}^{2}\right] .
\end{aligned}
$$

If $d_{2 n+1}>d_{2 n}$, inequality (34) implies

$$
d_{2 n+1}^{2} \leq \frac{3 c_{2}}{2-2 c_{1}-c_{2}} d_{2 n}^{2},
$$

a contradiction since

$$
\frac{3 c_{2}}{2-2 c_{1}-c_{2}}<1
$$

Thus $d_{2 n+1} \leq d_{2 n}$ and inequality (3.4) implies that

$$
d_{2 n+1}=d\left(y_{2 n+1}, y_{2 n+2}\right) \leq h d\left(y_{2 n}, y_{2 n+1}\right)=d_{2 n},
$$

where

$$
h^{2}=\frac{2 c_{1}+3 c_{2}}{2-c_{2}}<1
$$

Similarly,

$$
d_{2 n}^{2}=\left[d\left(y_{2 n}, y_{2 n+1}\right)\right]^{2}=\left[d\left(A x_{2 n}, B x_{2 n-1}\right)\right]^{2} \leq c_{1} \max \left\{d_{2 n-1}^{2}, d_{2 n}^{2}\right\}+c_{2}\left(\frac{3}{2} d_{2 n-1}^{2}+\frac{1}{2} d_{2 n}^{2}\right)
$$

and it follows as above that

$$
d_{2 n}=d\left(y_{2 n}, y_{2 n+1}\right) \leq h d\left(y_{2 n-1}, y_{2 n}\right)=d_{2 n-1}
$$

Consequently,

$$
d\left(y_{n+1}, y_{n}\right) \leq h d\left(y_{n}, y_{n-1}\right),
$$

for $n=1,2, \ldots$ Since $h<1$, this last inequality implies that $\left\{y_{n}\right\}$ is a Cauchy sequence in $X$

Now by replacing commuting mappings with compatible mappings, and by using four mappings as opposed to three, we prove the following generalization of the amended Theorem 11 which shows that only one of the mappings in Theorem 11 needs to be continuous 
THEOREM 3.1. Let $A, B, S$ and $T$ be self-mappings on a complete metric space $(X, d)$ satisfying conditions ( 31 ) and (3 2) and suppose that one of the mappings is continuous If $A$ and $B$ are compatible with $S$ and $T$ respectively, then $A, B, S$ and $T$ have a common fixed point $z$ Further, $z$ is the unique common fixed point of $A$ and $S$ and of $B$ and $T$

PROOF. Since $X$ is complete, it follows from Lemma 31 that the sequence $\left(\begin{array}{ll}3 & 3\end{array}\right)$ converges to a point $z$ in $X$. The subsequences $\left\{A x_{2 n}\right\},\left\{S x_{2 n}\right\},\left\{B x_{2 n-1}\right\}$ and $\left\{T x_{2 n-1}\right\}$ must therefore also converge to $z$

Let us first of all suppose that $S$ is continuous Since $A$ and $S$ are compatible, Proposition 21 implies that $\left\{S^{2} x_{2 n}\right\}$ and $\left\{A S x_{2 n}\right\}$ converge to $S z$ Using inequality (3 2) we have

$$
\begin{aligned}
& {\left[d\left(A S x_{2 n}, B x_{2 n-1}\right)\right]^{2} \leq c_{1} \max \left\{\left[d\left(S^{2} x_{2 n}, A S x_{2 n}\right)\right]^{2},\left[d\left(T x_{2 n-1}, B x_{2 n-1}\right)\right]^{2},\left[d\left(S^{2} x_{2 n}, T x_{2 n-1}\right)\right]^{2}\right\}} \\
& \quad+c_{2} \max \left\{d\left(S^{2} x_{2 n}, A S x_{2 n}\right) d\left(S^{2} x_{2 n}, B x_{2 n-1}\right), d\left(A S x_{2 n}, T x_{2 n-1}\right) d\left(B x_{2 n-1}, T x_{2 n-1}\right)\right\} \\
& \quad+c_{3} d\left(S^{2} x_{2 n}, B x_{2 n-1}\right) d\left(T x_{2 n-1}, A S x_{2 n}\right) .
\end{aligned}
$$

Letting $n$ tend to infinity we have

$$
[d(S z, z)]^{2} \leq\left(c_{1}+c_{3}\right)[d(S z, z)]^{2}
$$

and since $c_{1}+c_{3}<1$, it follows that $S z=z$

Using inequality (3 2) again we have

$$
\begin{aligned}
{\left[d\left(A z, B x_{2 n-1}\right)\right]^{2} \leq } & c_{1} \max \left\{[d(S z, A z)]^{2},\left[d\left(T x_{2 n-1}, B x_{2 n-1}\right)\right]^{2},\left[d\left(S z, T x_{2 n-1}\right)\right]^{2}\right\} \\
& +c_{2} \max \left\{d(S z, A z) d\left(S z, B x_{2 n-1}\right), d\left(A z, T x_{2 n-1}\right) d\left(B x_{2 n-1}, T x_{2 n-1}\right)\right\} \\
& +c_{3} d\left(S z, B x_{2 n-1}\right) d\left(T x_{2 n-1}, A z\right) .
\end{aligned}
$$

Letting $n$ tend to infinity we have

$$
[d(A z, z)]^{2} \leq c_{1}[d(S z, A z)]^{2}=c_{1}[d(z, A z)]^{2}
$$

and since $c<1$, it follows that $A z=z$.

Now since $A(X) \subseteq T(X)$, there exists a point $u$ in $X$ such that $z=A z=S z=T u$. Then on applying inequality (3 2$)$ to $[d(A z, B u)]^{2}$, it follows that

$$
[d(A z, B u)]^{2}=[d(z, B u)]^{2} \leq c_{1}[d(z, B u)]^{2},
$$

which implies that $z=B u$. Since $B$ and $T$ are compatible and $T u=B u=z$, we have from Proposition (2.1) that $T B u=B T u$ which implies that $T z=B z$. Again, on applying inequality (3.2) to $[d(A z, B z)]^{2}$, it follows that

$$
[d(A z, B z)]^{2}=[d(z, B z)]^{2} \leq\left(c_{1}+c_{3}\right)[d(z, B z)]^{2}
$$

and so $z=B z=T z$. We have therefore proved that $z$ is a common fixed point of $A, B, S$ and $T$ if $S$ is continuous.

The same result of course holds if we suppose that $T$ is continuous instead of $S$

Now suppose that $A$ is continuous. Since $A$ and $S$ are compatible, it follows from Proposition (2.1) that the sequences $\left\{A^{2} x_{2 n}\right\}$ and $\left\{S A x_{2 n}\right\}$ converge to $A z$ From inequality (32) we have

$$
\begin{aligned}
& {\left[d\left(A^{2} x_{2 n}, B x_{2 n-1}\right)\right]^{2} \leq c_{1} \max \left\{\left[d\left(S A x_{2 n}, A^{2} x_{2 n}\right)\right]^{2},\left[d\left(T x_{2 n-1}, B x_{2 n-1}\right)\right]^{2},\left[d\left(S A x_{2 n}, T x_{2 n-1}\right)\right]^{2}\right\}} \\
& +c_{2} \max \left\{d\left(S A x_{2 n}, A^{2} x_{2 n}\right) d\left(S A x_{2 n}, B x_{2 n-1}\right), d\left(A^{2} x_{2 n}, T x_{2 n-1}\right) d\left(B x_{2 n-1}, T x_{2 n-1}\right)\right\} \\
& \quad+c_{3} d\left(S A x_{2 n}, B x_{2 n-1}\right) d\left(T x_{2 n-1}, A^{2} x_{2 n}\right) .
\end{aligned}
$$

Letting $n$ tend to infinity we have

$$
[d(A z, z)]^{2} \leq\left(c_{1}+c_{3}\right)[d(A z, z)]^{2}
$$

and since $c_{1}+c_{2}<1$, it follows that $A z=z$. 
Now since $A(X) \subseteq T(X)$, there exists a point $v$ in $X$ such that $T v=A z=z \quad$ Using inequality (3 2), we have

$$
\begin{aligned}
{\left[d\left(A^{2} x_{2 n}, B v\right)\right]^{2} \leq } & c_{1} \max \left\{\left[d\left(S A x_{2 n}, A^{2} x_{2 n}\right)\right]^{2},[d(T v, B v)]^{2},\left[d\left(S A x_{2 n}, T v\right)\right]^{2}\right\} \\
& +c_{2} \max \left\{d\left(S A x_{2 n}, A^{2} x_{2 n}\right) d\left(S A x_{2 n}, B v\right), d\left(A^{2} x_{2 n}, T v\right) d(B v, T v)\right\} \\
& +c_{3} d\left(S A x_{2 n}, B v\right) d\left(T v, A^{2} x_{2 n}\right) .
\end{aligned}
$$

Letting $n$ tend to infinity, we have

$$
[d(A z, B v)]^{2}=[d(z, B v)]^{2} \leq c_{1}[d(z, B v)]^{2},
$$

which implies that $z=B v$ Since $B$ and $T$ are compatible and $T v=B v=z$, it follows from Proposition (2 1) that $T B v=B T v$ which implies that $T z=B z \quad$ Similarly, applying inequality (3 2) to $\left[d\left(A x_{2 n}, B z\right)\right]^{2}$, it follows that $T z=B z=z$

Since $B(X) \subseteq S(X)$, there exists point $w$ in $X$ such that $S w=B z=z$ Using inequality (3 2), we have

$$
[d(A w, z)]^{2}=[d(A w, B z)]^{2} \leq c_{1}[d(S w, A w)]^{2}=c_{1}[d(z, A w)]^{2}
$$

and it follows that $z=A w$ Since $A$ and $S$ are compatible and $A w=S w$, it follows from Proposition (2 1) that $A S w=S A w$ and so $A z=S z \quad$ Thus $z$ is a common fixed point of $A, B, S$ and $T$ if $A$ is continuous

The same result holds if we suppose that $B$ is continuous instead of $A$

Finally, let us suppose that $A$ and $S$ have a second common fixed $z^{\prime} \quad$ Then from inequality (3 2) we have

$$
\begin{aligned}
{\left[d\left(z^{\prime}, z\right)\right]^{2}=} & {\left[d\left(A z^{\prime}, B z\right)\right]^{2} \leq c_{1} \max \left\{\left[d\left(S z^{\prime}, A z^{\prime}\right)\right]^{2},[d(T z, B z)]^{2},\left[d\left(S z^{\prime}, T z\right)\right]^{2}\right\} } \\
& \quad+c_{2} \max \left\{d\left(S z^{\prime}, A z^{\prime}\right) d\left(S z^{\prime}, B z\right), d\left(A z^{\prime}, T z\right) d(B z, T z)\right\}+c_{3} d\left(S z^{\prime}, B z\right) d\left(T z, A z^{\prime}\right) \\
= & \left(c_{1}+c_{3}\right)\left[d\left(z^{\prime}, z\right)\right]^{2},
\end{aligned}
$$

and it follows that $z=z^{\prime}$, since $c_{1}+c_{3}<1$ Analogously, $z$ is the unique common fixed point of $B$ and $T$ This completes the proof of the theorem.

We now prove the following common fixed point theorem for a compact metric space

THEOREM 3.2. Let $(X, d)$ be a compact metric space and let $A, B, S$ and $T$ be continuous mappings of $X$ into itself satisfying the conditions

$$
A(X) \subseteq T(X) \text { and } B(X) \subseteq S(X),
$$

$A$ and $B$ compatible with $S$ and $T$ respectively

and the inequality

$$
\begin{aligned}
{[d(A x, B y)]^{2}<} & c \\
+ & \max \left\{[d(S x, A x)]^{2},[d(T y, B y)]^{2},[d(S x, T y)]^{2}\right\} \\
& +\frac{1}{2}(1-c) \max \{d(S x, A x) d(S x, B y), d(A x, T y) d(B y, T y)\} \\
& +(1-c) d(S x, B y) d(T y, A x)
\end{aligned}
$$

for all $x, y$ in $X$ for which the right hand side of (3.7) is positive, where $0<c<1$ Then $A, B, S$ and $T$ have a common fixed point $z$. Further, $z$ is the unique common fixed point of $A$ and $S$ and of $B$ and $T$

PROOF. Suppose first of all that there exists $c_{1}, c_{2}, c_{3} \geq 0$, with $c_{1}+2 c_{2}<1$ and $c_{1}+c_{3}<1$ such that inequality (3.2) is satisfied. Then the result follows from Theorem 31 .

Now suppose that no such $c_{1}, c_{2}, c_{3}$ exist. Then if $\left\{a_{n}\right\},\left\{b_{n}\right\}$ and $\left\{c_{n}\right\}$ are monotonically increasing sequences of real numbers converging to $c, \frac{1}{2}(1-c)$ and $(1-c)$ respectively, we can find sequences $\left\{x_{n}\right\}$ and $\left\{y_{n}\right\}$ in $X$ such that 


$$
\begin{aligned}
{\left[d\left(A x_{n}, B y_{n}\right)\right]^{2}>} & a_{n} \max \left\{\left[d\left(S x_{n}, A x_{n}\right)\right]^{2},\left[d\left(T y_{n}, B y_{n}\right)\right]^{2},\left[d\left(S x_{n}, T y_{n}\right)\right]^{2}\right\} \\
& +b_{n} \max \left\{d\left(S x_{n}, A x_{n}\right) d\left(S x_{n}, B y_{n}\right), d\left(A x_{n}, T y_{n}\right) d\left(B y_{n}, T y_{n}\right)\right\} \\
& +c_{n} d\left(S x_{n}, B y_{n}\right) d\left(T y_{n}, A x_{n}\right)
\end{aligned}
$$

for $n=1,2, \ldots \quad$ Since $X$ is compact we may assume, by taking subsequences if necessary, that the sequences $\left\{x_{n}\right\}$ and $\left\{y_{n}\right\}$ converge to $x$ and $y$ respectively Letting $n$ tend to infinity in inequality (3 8) we have, since $A, B, S$ and $T$ are continuous,

$$
\begin{aligned}
{[d(A x, B y)]^{2} \geq } & c \\
& \max \left\{[d(S x, A x)]^{2},[d(T y, B y)]^{2},[d(S x, T y)]^{2}\right\} \\
& +\frac{1}{2}(1-c) \max \{d(S x, A x) d(S x, B y), d(A x, T y) d(B y, T y)\} \\
& +(1-c) d(S x, B y) d(T y, A x) .
\end{aligned}
$$

This is only possible if $A x=B y=S x=T y$ Since $A$ and $S$ are compatible and $A x=S x$, it follows from Proposition (2 1) that $A S x=A^{2} x=S A x$

Suppose that $A^{2} x \neq B y$ Then using inequality (3 7) we have

$$
\begin{aligned}
{\left[d\left(A^{2} x, B y\right)\right]^{2}<c } & \max \left\{\left[d\left(S A x, A^{2} x\right)\right]^{2},[d(T y, B y)]^{2},[d(S A x, T y)]^{2}\right\} \\
& +\frac{1}{2}(1-c) \max \left\{d\left(S A x, A^{2} x\right) d(S A x, B y), d\left(A^{2} x, T y\right) d(B y, T y)\right\} \\
& +(1-c) d(S A x, B y) d\left(T y, A^{2} x\right) \\
= & \left.d\left(A^{2} x, B y\right)\right]^{2},
\end{aligned}
$$

a contradiction and so $A^{2} x=B y=A B y$. It follows that $B y=z$ is a fixed point of $A$ Further,

$$
S z=S B y=S A x=A S x=A B y=A z=z
$$

and it follows that $z$ is a common fixed point of $S$ and $A$

Similarly, we can prove that $B$ and $T$ have a common fixed point $v$ If $z \neq v$, then on using inequality (3.7) we have

$$
\begin{aligned}
{[d(z, v)]^{2}=} & {[d(A x, B v)]^{2} } \\
< & c \max \left\{[d(S z, A z)]^{2},[d(T v, B v)]^{2},[d(S z, T v)]^{2}\right\} \\
& \quad+\frac{1}{2}(1-c) \max \{d(S z, A z) d(S z, B v), d(A z, T v) d(B v, T v)\} \\
& \quad+(1-c) d(S x, B v) d(T v, A z) \\
= & {[d(z, v)]^{2} }
\end{aligned}
$$

a contraction. Thus $z$ is a common fixed point of $A, B, S$ and $T$. It follows easily that $z$ is the unique fixed point of $A$ and $S$ and of $B$ and $T$.

Finally, note that some obvious corollaries can be obtained from Theorems 31 and 3.2 by letting $A=B$ and $S=T$.

\section{REFERENCES}

[1] JUNGCK, G., Compatible mappings and common fixed points, Internat. J. Math. Math. Scl. 9 (1986), 771-773.

[2] SESSA, S., On a weak commutativity condition of mappings in fixed point considerations, Publ. Inst. Math. 32 (1982), 149-153.

[3] SHARMA, B. K. and SAHU, N. K., Common fixed points of three continuous mappings, Math. Student 59 (1991), 77-80 


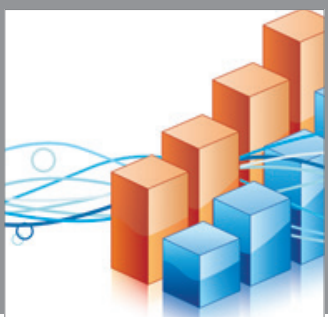

Advances in

Operations Research

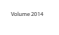

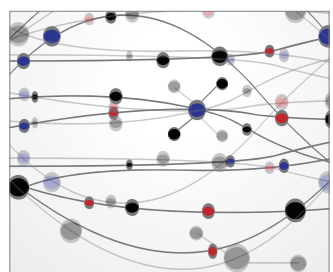

\section{The Scientific} World Journal
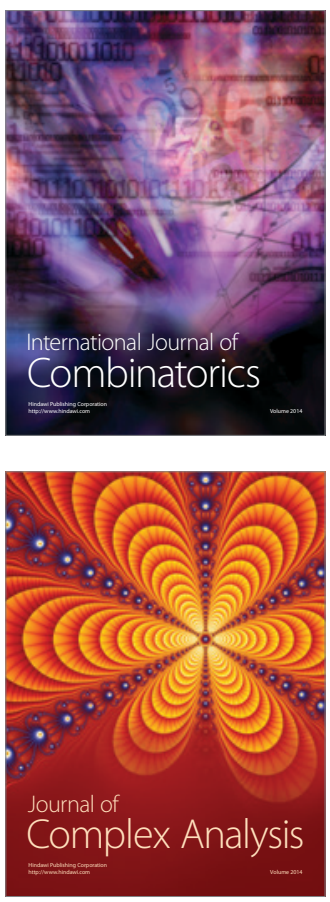

International Journal of

Mathematics and

Mathematical

Sciences
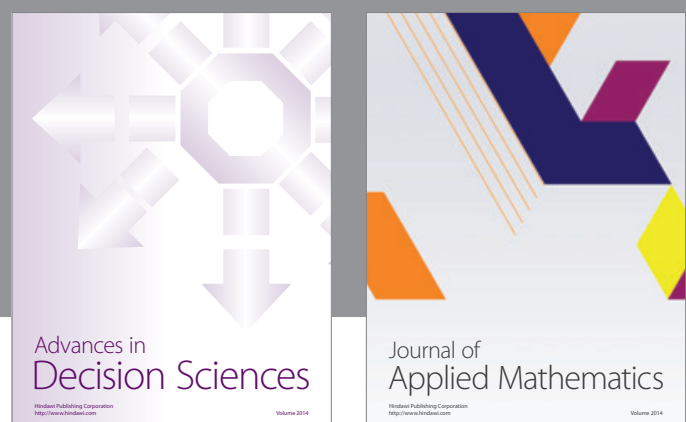

Journal of

Applied Mathematics
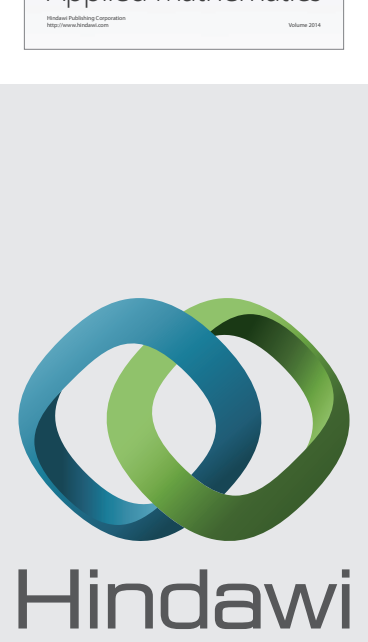

Submit your manuscripts at http://www.hindawi.com
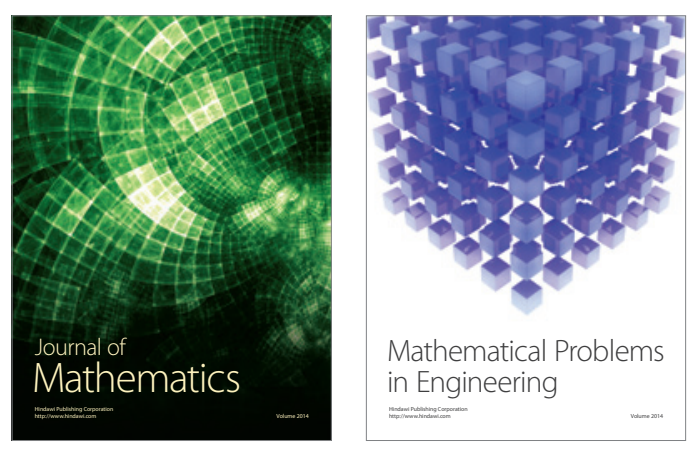

Mathematical Problems in Engineering
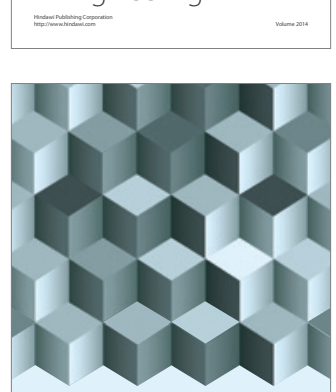

Journal of

Function Spaces
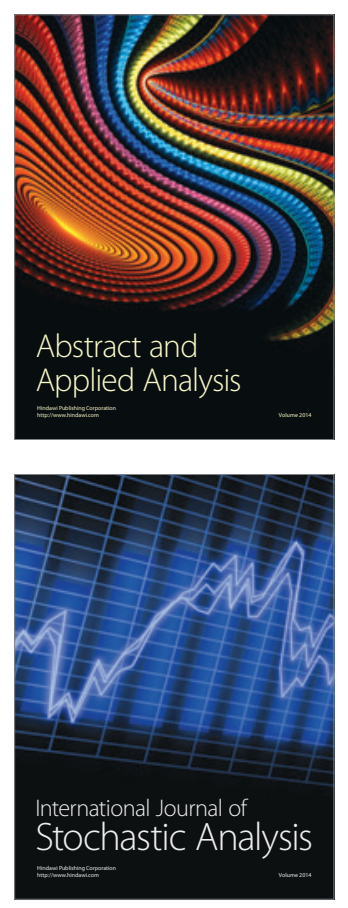

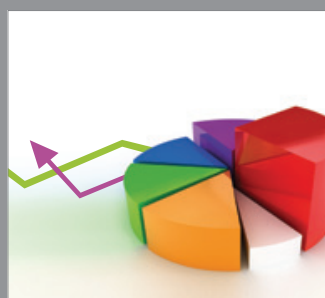

ournal of

Probability and Statistics

Promensencen
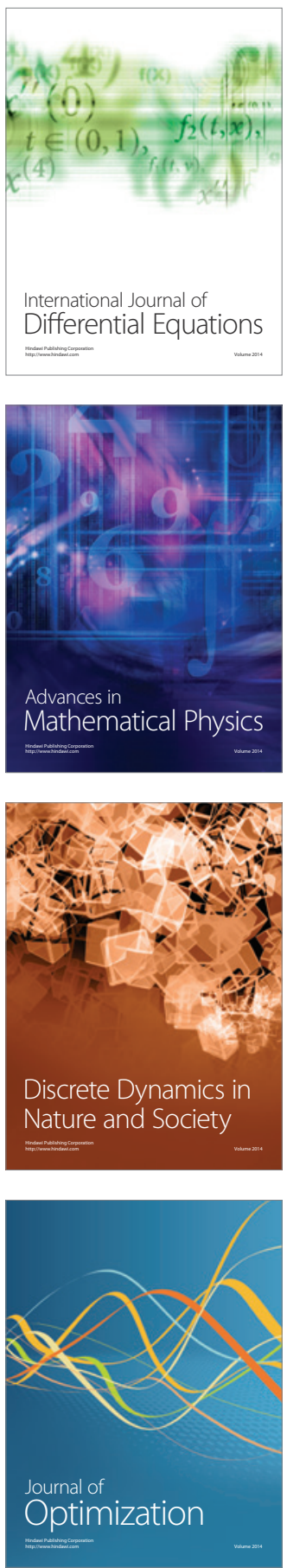